drug than the potassium salt; but, he continued, the strontium salt is superior to either the sodium or potassium salt, and for several years he has been recommending the strontium salt above all others.

Dr. W. E. Ronertson, Philadelphia, called attention to the work on diseases of the heart written by Napoleon's physician, Corvisart, in which Napoleon's symptoms are described in such a way as to lead to no more definite conclusion than that his was a case of bradycardia, and probably to-day, instead of receiving bromids, as Dr. Robinson suggests, he would have been subjected to a very careful system of cardio-vascular tracings, where it would probably end.

DR. W. IV. Tompkins, Charleston, W. Va,, was convinced that the bromid of potassium is the most toxic of the bromids. Dr. Robinson's paper, he said, went very exhaustively into the use of the bromid of strontium, except that he failed to mention its use in cases of indigestion. In some of these cases it is largely used combined with aromatics, such as peppermint water. Dr. Tompkins thought that there is not very much individual opinion exercised on the part of the individual pre: scriber. He is so apt to give only those prescriptions that appear in the text-book. He believes, further, that there is a decided idiosyncrasy in bromids, particularly, and that one can get decided beneficial effects with small doses thoroughly diluted. They are also less dangerous than large doses.

Dr. C. B. Lowe, Germantown, Pa., was under the impression that the ill effect from potassium salts is because they act on the heart musele as strong depressants, and that that is one of the reasons why sodium salts are usually selected.

Dr. W. J. Robrsson, New York, City, emphasized the superiority of strontium bromids over potassium bromid. Through force of habit, he said, whenever physicians think of bromid, they think of potassium bromid, and therefore he emphasized the dangers attending its use. He mentioned the fact that strontium bromid acts as a gastric antiseptic, and it is very good in so-called nervous dyspepsia. Dr. Robinson replied to Dr. Lowe that Dr. Bennion reports that they treated one class of their epileptics with mixed bromids and the other class with strontium bromid only, and they reached the conclusion that the strontium bromid is superior to the mixed bromids.

\section{THE INFIUUENCE OF THE DUCTLESS GLANDS OVER METABOLISM,}

\section{WITH ILLESTRATIVE CASES.*}

LEO M. CRAF'TS, B.L., M.D.

Professor of Nervous and Mental Diseases in the Medical Department of Hamline University ; Visiting Neurologist to Minneapolis City Hospital, Asbury Methodist, St. Barnabas and Swedish Hospitals. MINNEAPOLIS.

The increasing elucidation of the functions of the ductless glands and their influence on the morphology of the human organism renders their study most fascinating. While much of the ground is still speculative, enough has been established to show that the thyroid is closely correlated in its action with the pituitary body, the adrenals and the thymus gland. And they must be considered together for a rational view in predicating near and remote effects on the functional activity of the organism, most of which have formerly been ascribed to the thyroid alone. The normal and full development of the individual, phrsical, nervous and mental, and the preservation of cellular activity, depend, in the ultimate, on the integrity of these organs.

Research, clinical observation and experiment have now fairly established that the parenchyma of the thy-

* Read in the séction on Nelvous and Mental Diseases of the American Medical Association, at the Fifty-eighth Annual Session, lisd at Atlantic City, June, 1907. roid supplies the blood with a secretion which, by selective application, promotes the activities of the anterior pituitary body. The investigations of Baumann, added to by Mabel!e and Ewald, go to show that iodin is probably the most active agent in thyroid secretion, with arsenic a subordinate, but still important element. It is d fficult, however, to reconcile all phenomena as entirely explained by the action of these metals alone, for their administration in ordinary forms does not give clinical results, such as we obtain from the use of throid material itself. The anterior and posterior pituitary bolies differ materially in structure. The posterior is chicfly neural, being made up of neuroglia. nerre cells and epithelial-cell tubules. The anterior is, in the main, glandular, being comprised of vesicular eavities, lined with epithelium and holding colloid material. These are enveloped in a rich mass of capillaries and lymphatics, strongly resembling throid structure. But, although there is a close relationship between them, the action of the pituitary is distinctly different from that of the thyroid. Only a light fibrous reticule separates the anterior from the posterior pituitary body and they share the same capillary and Jymph supply.

As shown by the work of Berkley, Hirshfeld and others, only srmpathetic nerre fibers articulate with the anterior pituitary structure. Through these filaments, coming from the cervical ganglionic supply by way of the carotid plexus, thence, directly through the cervicothoracic sympathetic chain, the splanchnics and the semilunar ganglia, the pituitary is linked with the adrenals. for the transmission of nerrous impulse, and stimulation of their glandular activity. Experiments with pituitary extracts by such observers as Schafer, Howell and Osborne, show clear evidence of stimulation of the adrenal bodies, and both experimental ablation of the cervical sympathetic in animals and the fatal results of their removal in the human subject further prove that the pituitary is the center of energy of the suprarenal glands. Rapid emaciation and death also follow the destruction of the pituitary or the severing of the neural path between these organs.

Further investigation has shown that the pituitary body depends on the thrroid in turn for its stimulus. So when the thyroid is acting in excess, theoretically, it overstimulates this organ, which in turn transmits its added excitation to the adrenals with increased production of the secretion of those organs poured into the blood. When, on the other hand, there is lowered functioning in the thyroid, the hypophysis lacks its normal stimulus, as is also the case when it is inherently weak, or when there is lowered oxidation of the blood from any cause, or when active toxics charge the blood stream.

Although the structure and association of the thyroid and the pituitary would perhaps seem to indicate that their functional product would be identical, close analysis shows that they are not the same, and that phosphorus is the chief element in the activity of the latter, and the fundamental part of both the osseous and nervous systems.

From the beginnings of life to full adolescence the thymus plays the most important rôle. Its evident office is to reinforce the hrpophysis during this time of excessive demand, and supplies the major part of the phosphorus to the rapidly building neural and bony structures. It also indirectly stimulates the adrenals in their work as well. Inherent weakness in the thymus, or anything that interferes with its action, impairs the organism's most vital development. In childhood it is 
normally the most active of all the ductless glands, the thyroids are next and the adrenals last; while in adult life the adrenals are foremost in importance. On this gland (the thymus) the normal unfolding of mentality depends, and statistics like those of Bourneville show that in the great majority of feeble-minded children, not cretins, the thymus is wanting or deficient. It has fulfilled its mission with the completion of adolescence.

The innervation of the suprarenal bodies from the pituitary through the direct channel of the cervicothoracic sympathetic chain has already been pointed out. The stimulation of their activities, then, depends on the integrity of the former gland, which in turn receives its impulsion from the presence of the thyroid sccretion, in normal amount and quality in the blood. In the parenchyma of the adrenals a colloid material, a chromogen, is formed and poured directly into the blood going to the inferior vena cava. Thence it reaches the right auricle, directly stimulates the heart muscle, has a remarkable affinity for oxygen and absorbs this gas in the pulmonary distribution as it floats in the blood plasma. The blood elements are bathed in the oxygenated medium, and the hemoglobin is changed again into oxyhemoglobin. This quality of the adrenal secretion makes it the great oxygenating agent of the organism, and is the final purpose of the great sistem, made up of these organs together with the thyroid and the pituitary bodies. They sustain the action of the heart, respiration, metabolism and cell nutrition. Overaction or defect of structure or function of any of this group of organs gives rise to one or another of a remarkable series of conditions.

The foregoing outline of the now fairly well-established physiologic action and correlations of these glandular structures opens the way to an analysis of the effects of disturbances in the balance and harmony of their activities. And the thyroid naturally claims attention first. In exophthalmic goiter the thyroid is supposably in a hyperactive condition and contributing an excess of secretion to the blood. This overstimulates the pituitary, which in turn excites the adrenals, resulting in an excess of its product being poured into the blood with hyperoxidation the ultimate result and the basis of the syndrome in the eartier stages, while the later manifestations, such as the emaciation, weakness, bronzing and depression, are indicative of insufficiency of the adrenals, exhausted through continued overstimulation.

Myxedema and cretinism naturally group together for consideration. They are the same in underlying causative factors, but occurring at different stages in organic life. Both of them illustrate the results of deficient oxygenation of tissues from failure of the adrenals, due to thyroid deficiency in proper stimulation of the pituitary. The one affects the mature adult, the other the growing child. Thyroid treatment in both simply supplies to the waiting pituitary the stimulus it needs for renewing its energizing power to the adrenals. And the anterior pituitary is the great energy center of the organism, as the posterior is its ultimate sensorium, where nervous shock and stress have their final effect.

The great solar plexus probably first takes the impact of severe nerve stress of all kinds, the waves of impulse traveling most directly and with greatest force to the hypophysis like an overcharge on an electric wire, while there is, of course, some general diffusion, the entire sensory sphere, and especially the central vasomotor supply, suffers from the sharp irritation: In this con- nection it. is interesting to note the bearing of thie effects. of sudden and profound.shacks, as well as the importance of general wear of the nerrous system from any cause, with its accompanying excess waste of phosphor elements and evidence of varying degrees of adrenal overstimulation or failure, with such symptoms as tachycardia, muscular weakness, tremors, pallor, conditions of mental exaltation and depression, and the interesting vasomotor relationship between the sexual organs, other erectile tissues and the thyroid. These will receive further consideration in connection with illustrative cases.

One more set of conditions will claim our attention. acromegaly and gigantism. These are analogous to the last two, i. e., myxedema and cretinism, but while the latter are due to deficient thyroid action, with resulting lowered oxygenation, the former are due to overactivity of the pituitary, stimulating the adrenals, with hyperoxidation as the result. The one occurs in youth, the other in mature life. Toxemias, persistence of the thrmus, adding to the phosphoric production-hypertrophy. of the pituitary often following-and actual new growths in this gland, are the provoking cause of this overstimulation of the adrenals and the excess of oxidation in the tissues.

Absence of the thymus results in mental deficiency. When such cases, also showing lack of phrsical derclopment, are given thyroid medication, they respond in body growth, but not in mental improvement, and the bones soften at the same time, for it is phosphorus that is needed for both the nerrous system and the bones rather than better oxidation, which is what thyroid stimulation gives.

To summarize, then, the present views of the functioning and results of disturbances of this group of organs: Exophthalmic goiter is overstimulation with altered function of the thyroid gland, with increased impulse from the pituitary through its sympathetic innerration of the adrenals, causing hyperoxidation in the organism by excess production of its secretion in the blood. On this later supervenes failure of those bodies from exhaustion.

Myxedema and cretinism are pure results of deficient oxidation from lack of thrroid activity or from innate lack of power in the pituitary due to insufficient phosphorus elements.

Acromegaly and gigantism result from overactivity of the pituitary acting on the adrenals, with abnormal hypernutrition.

Absence of the thymus always predicates deficient mentality in the subject. Its persistence beyond the time when its activity should cease contributes an excess of phosphorus and its results.

Various systemic conditions show clear indications of lack of sufficient oxidation or of phosphorus in the individual, but without going to the point of the actual picture of any of the above states. Chlorosis and other forms of anemia with diminished hemoglobin, pallor and lowered strength show a degree of failure of the adrenals. The various functional neuroses like neurasthenia, or hysteria, with their tremors, excessive perspiration, diminished mental power, easy fatigue, tachycardia and polyuria, point distinctly to lack both of phosphorus and oxygen radicals in the tissues and body fluids from weakened action of the pituitary and the adrenals, and thyroid functioning is probably also implicated. Some things, too, in the behavior of the thrroid, in goiter, fail of satisfactory explanation on the 
theory simply of overstimulation in the early stages. It does not explain the fact that medication with thyroid extract in many cases in this stage is signally beneficial. And there is reason to conclude either that there is rather a change in the gland function or that the secretion is altered in composition, becoming toxjc, with resulting irritation of the hypophysis, and that thyroid extract contributes the normal agent. The adrenals may, in a measure, be stimulated from other sources than its innervation, and iron and arsenic are of value for this purpose. The iron plays a double rôle, helping to increase the oxygen-carrying capacity of the blood as well as stimulating the oxygenating power from the adrenals.

CASE 1.-History.-A man, aged 43, of good family and previous health, came under observation on Dec. 24, 1902. Four months before he had what was called a "boil" on the left side of his neck over the left lobe of the thyroid. It was lanced and had continued to discharge freely, pus and a glary fluid. Another opening occurred at the supra-sternal notch, and also one further round on the left side of the neck. All three were connected by sinuses. Irrigation had been kept up all the time by his physician. He was troubled with cold hands and feet, pain in the lower legs, and sweating and hot flashes on the right side of the body. Appetite good, bowels regular. He had been melancholy and much depressed for the past three weeks, with crying spells, and he had lost much flesh.

The left side of the neck showed livid discoloration, the normal outline flattened, and the surface indurated. There were three sinus openings. The pulse was weak and slightly rapid. The left lobe and isthmus of the thyroid had evidently been largely destroyed. $\mathrm{He}$ was not getting sufficient thyroid action, and oxidation was evidently suffering. The sinuses were allowed to heal at once.

Treatment.-He was put on such general tonies as organic iron and manganese, and thyroid extract in two-grain doses was given three times a day. Improvement began promptly. $\mathrm{He}$ was under observation for about two months. The melancholic condition entirely cleared up, he gained flesh, and the color, which had been dusky, became normal. He went home to South Dakota, continued to use the thyroid for some time longer and has remained perfectly well.

CASE 2.-History.-A young woman, aged 22, seen in June, 1903 , of a very interesting neurologic family history. Maternal grandmother living and has goiter and edema of legs. Maternal grandfather had kidney trouble. Paternal grandmother died of asthma, paternal grandrather was rheumatic. Maternal granduncle had some form of paraplegia. A maternal aunt had goiter, another had anemia, goiter and "sick headaches." A third maternal aunt has a goiter, and another is subject to "fainting spells." Still another had an attack of delirium. Three paternal aunts died of rapid tuberculosis. A maternal cousin died in convulsions and another with chorea. The patient's mother is anemic, has numbness in the right leg, muscular cramps and "sinking spells." The subject herself was frail till 3 years old, rugged from then till two years before coming under control. Then began to feel weak and tired, with peculiar precordial feeling. She noticed beginning enlargement of the neck at the same time. There was insomnia, bowels regular. Prominence of the eyes was noticed. Severe attacks of headache began six months before, sometimes lasting for days. Menstruation normal. Was doing heavy lifting at this time. Free perspiration and easy flushing. She was well developed and nourished, but had a peculiar nallor, a marked goiter, the neck measuring seventeen and onehalf inches. Pulse 100, bounding and irritable, slight tremor and very slight prominence of the eyes. There was an area of paresthesia on the right thigh, quite marked edema at times, of the lower legs, and marked general sensitiveness of the body surface.

Treatment.-She was put on thyroids, unguentum hydrargyri oxidi rubrum, galvanism over the gland, the cervical ganglia and the precordium thirty to forty minutes three times a week, and a combination of strophanthus, digitalis and nitroglycerin. There was great difficulty for a time in controlling the attacks of head pain, which had the appearance of being extreme. They were accompanied by writhing and twitching, which had an almost involuntary look, and there was also much cramping of the legs in the attacks. The relationship between these attacks and meteorological changes was very striking.

She was under continuous observation for a year and a quarter. Improvement in all conditions was fairly constant. The goiter diminished in size, the edema improved, the heart action steadied. Gelsemin in 1/134 gr. doses every hour, controlled the head pain most successfully. These attacks came further apart. Strength and endurance improved: The pallor gave way, and she began taking on flash. Thyroid medication was constant. When dismissed the circumference of the neek had diminished over three inches, and only the isthmus of the gland remained slightly prominent. The heart was normal, the head pain had ceased, she had gained 30 pounds. She has since remained well.

This was a clear case of exophthalmic goiter, with some hysteric coloring. The exophthalmic goiter was evidently in a stage of orerstimulation from the thyroid. Theoretically, thyroid medication was contraindicated, but she was kept on full doses for a period of over one and one-half years, with perfect results, and so far $I$ have not seen a single case aggravated by its use.

CASE 3.-History.-Young lady, aged 24, first seen in February, 1905. Her father was a melancholic and committed suicide. He was also a heavy drinker. Patient has never been strong. Always gloomy, inclined to worry. Did not mature till 17. Had a "blue spell" then, with suicidal impulse, again at 18 , and again at 20 . Insomnia during the attacks. Began to be despondent a month before coming under treatment. Suicidal impulse. Gloom and abstraction. She was pale and had a depressed expression, was poorly nourished, pulse rather weak and nervous manner, sluggish bowels and sleeping poorly.

Treatment.-She was put on such nerve sedatives as codein phosphate, the valerinates, hypnotics, and nucleins, with heart tonics. Her condition continued in an indifferent way, with slight improvement for some months. In June, 1905 , she was put on full thyroid feeding. She immediately began to gain in a remarkable way in general condition, to feel more cheerful, better strength, and putting on flesh. Improvement continued steadily and in about five months she had gained 25 pounds, was in normal mental state, except for the fear that the trou. ble might come back. Her color did not improve with the gain in flesh, and instead took on a peculiar waxy white look. With the use of organic iron and the phosphates, her color became good.

The peculiar pallor shown in this case has been noterl several times in patients on thyroid feeding and is difficult of explanation.

Case 4.-History.-Arthur B., a boy, 15 years old, was first seen June 27, 1904. Nervous temperament and rheumatism on father's side. About one and a half years ago he began to be restless, made occasional peculiar remarks, and was careless in his habits. These symptoms disappeared in a short time, similar ones recurring at intervals. $\mathrm{He}$ is unusually bright in school, has a ravenous appetite, and does everything in a hurry. He is sturdy looking, well developed and nourished. He is restless and it is difficult to hold his atten. tion. Heart action and pulse normal. Slight reflex and myotatic irritability. Inclined to be surly. He proved rather hard to control, as he was determined to be too active.

Treatment.-Varying combinations of medication were resort. ed to at times, but the main lines were the use of codein phos. phate, trional, nucleins and lecithin, and other forms of phos. phorus. Thyroid feeding was begun at once and kept the most constant element of the treatment. He did not do as well when it was suspended. And when it was combined with some 
form of phosphorus he made the best progress. With some variations he made good gain and reached full recovery after a year and a half of attention, and resumed his school work and all other normal activities.

CASE 5.-History-A girl of 22, unmarried; considerable neurophatic taint on the mother's side. The patient was well as a child. Three years ago was overtaxed. Became hrperesthetic, easily startled; dysmenorrhea came on. Marked insomnia, headache, poor memory, easily fatigued. Dysentery for six weeks a year ago, and vertigo. She had nerrous "spells" with screaming, palpitation and numb feelings in a hand or foot at times. She was fairly well developed and nourished, pale, nervous manner, reflex irritability. Left eye prominent, with dilated and irregular pupil-old injury, heart regular, pulse 76 . Tremor of hands and thyroid slightly prominent.

Treatment.-She was put on accepted lines of control, with ordinary medication, massage, electricity, and the like, making only fairly good progress. After about six months she was put on thyroid feeding, experimentally, with other conditions going on just as before. She soon began to gain rapidly in every way. The capricious appetite became better, she slept well. The nervous storms came at much longer intervals and then ceased. Her nervous tolerance improved, she grew stronger, and began to take on flesh rapidly, gaining over 30 pounds in a few months. The thyroids were then suspended at intervals and reinstituted, always with distinct results. This case also showed the peculiar pallor referred to in another case.

Case 6.-History:-A young girl of 17 , seen first in Norember, 1906. There was no material nervous taint. She had never been rugged. About a vear ago received a strain of the abdominal muscles; had been studying hard, began to run down, became very restless and fretful, sleeping poorly, constant gnawing of the finger nails. She was very slight, exceedingly restless, with a frightened manner and expression, crying easily, and very exeitable. Pulse somewhat rapid and weak.

Treatment.-She was put on hypnotics, phosphorus combinations, arsenic and thyroid feeding at once. Improvement followed with striking rapidity, the nervous agitation subsiding; she began to sleep and eat well, complete recovery of strength and nervous tone followed, with good gain in general nutrition and flesh, although not as notable an amount as in some other cases.

These illustrative cascs have been selected to show the application of the deductions in the foregoing part of the paper. Case 1, with partial destruction of the thyroid gland, showed the clear syndrome of adrenal incompetence with deficient oxrgenation. The result of thyroid feeding was complete and prompt, and enough of the gland remained to make it permanent. Case 2 was a characteristic one of exophthalmic goiter. with added hysteric coloring. The goiter was undoubtedly in the stage of so-called overstimulation, yet the thyroid feeding gave the happiest. result. This case also shows the remarkable effect as a nutrient tonic which it posseses in common with thymus extract. Case 3 , one of simple melancholia, shows the indications of suprarenal inadequacy with lowered oxygenation present in other conditions than the organic ones usually considered, and illustrates the value of stimulating this particular glandular system. The power over nutrition is also powerfully shown in this case. Cases 4 and 6 , on the borderland of developmental insanity, illustrate its bearing in the conditions of threatening failure of mental evolution. In these cases phosphorus from the pituitary and thymus plays as important part as the thyroid with its iodin, for in these cases the thymus is presumably-more at fault, and is not supplying its phosphor product fully enough to meet the demands of the ranidly dereloping nervous system. Case 5, one of hysteroneurasthenia, slows the evident lack of functioning activity in the thyroid-pituitary-adrenal groun. In these cases there is a vicious circle. The condition of nerve exhaustion is accompanied by excessive waste of phosphorus elements, the. lowered tone diminishes the adrenal stimulus, and the oxidizing power, with its lessened nutrition of all structures and autointoxication is present. Thyroids and lecithin elements are indicated here, as they are both needed to renew the activity first of the pituitary body, the great energy center of the organism, and through it to increase the secretory activity of the adrenals and the part they play in sustaining the great vital functions.

\section{DISCLSSION}

Dr. J. Madisor Taylor, Philadelphia, said that one point. that Dr. Crafts in all probability did not have time to bring out is the fact that these glands are classed with organisms which are regarded as primary - the lungs, heart and liverand that the functions extend throughout the whole length and breadth of the body. The applicability of this principle is very much larger than the cases described by Dr. Crafts, relating largely to the organs of this particular character. The point which Dr. Taylor emphasized is that through a theory which we can not understand this group of organs, which Dr. Crafts has outlined so well, controls practically the whole of the body organisms, more particulary in dealing with the volume of tissue regulation through the volume of the blood, and the regulation of the depressed functional cavities through the bondy.

\section{ACID ATTOINTOXICATION AND SYSTEMIC DISEASE, THE CAUSE OF EROSION AND ABRASION.* \\ EUGENE S. TALBOT, M.S., D.D.S., M.D., LJ.D. CHICAGo.}

The prevailing opinion has been, and is taught to-dar, that the cause of diseases of the mouth, jaws and teeth are of Iocal origin. Peculiarly is this true of erosion and abrasion, judging from the literature. It has ben my purpose in life to devote my time to researches to disabuse the minds of members of the profession of this dormant notion. While it is possible that local factors may enter into the etiology, yet the great factor in all cases of dental pathology is constitutional rather than local. In this paper I shall furnish another proof of these assertions. No one factor, either local or constitutional, can be accepted as the cause of a given disease. Thus, tooth decay is due to lactic acid ferment; the great cause, however, is evolution and degeneration. Outside of the etiology of tooth decay perhaps no subject has caused so much anxiety to the members of the profession as that of erosion and abrasion. The two terms were used by early writers to designate the destruction of the tooth or teeth, erosion being a gractual wearing away of the grinding surfaces of the teeth, abrasion the destruction of the surface of the teeth in connection with the mucous membranes of the mouth. The two terms emploved in the light of my researches really mean one and the same thing, as I shall demonstrate later. It is well, however, to retain the two terms because they indicate to a certain extent the location of tooth destruction.

In my papers. "Pulp Degeneration."1 "Constitutional Causes of Tooth Decar." "Constitutional Causes

* Tead in the Section on Stomatology of the American Medical Assuciation at the Fifty-eighth Annual Session. Inne, 1907.

1. The Jocknal A. M. A., Allg. 6, 1904, p. 385

2. Dental Digest, December, 1903 . 